\title{
ROOM-TEMPERATURE WAFER-LEVEL VACUUM SEALING BY COMPRESSION OF HIGH-SPEED WIRE BONDED GOLD BUMPS
}

\author{
M. Antelius, A. C. Fischer, N. Roxhed, G. Stemme and F. Niklaus \\ KTH Royal Institute of Technology, Stockholm, SWEDEN
}

\begin{abstract}
This paper reports experimental results of a novel room temperature vacuum sealing process based on compressing wire bonded gold "bumps", causing a material flow into the access ports of vacuum-cavities. The leak rate out of manufactured cavities was measured over 5 days and evaluated to less than the detection limit, $6 \times 10^{-12}$ $\mathrm{mbarL} / \mathrm{s}$, per sealed port. The cavities have been sealed at a vacuum level below 10 mbar. The method enables sealing of vacuum cavities at room temperature using standard commercial tools and processes.
\end{abstract}

\section{KEYWORDS}

vacuum sealing, packaging, wire bonding, room temperature, MEMS

\section{INTRODUCTION}

There is a need for low-cost and reliable vacuum encapsulation processes for a wide range of MEMS devices, such as infrared sensors, accelerometers and gyroscopes [1]. For sensitive devices, sealing at room temperature can be vital. In addition, the low temperature can reduce out-gassing from surfaces which in turn will reduce the need for getters, necessary to obtain low vacuum pressures. Hermetic packaging at room temperature is challenging since few bonding methods yield truly hermetic seals at room temperature. Reported methods include plasma activated direct bonding [2] and local heating of sealing structures [3]. However, these methods require certain material systems for the activation to function or tailor-made and fairly complex sealing structures.

Low temperature deformation of gold has recently been used for both packaging and micro-structuring applications. The possibility to hermetically seal liquid filled cavities at room temperature and ambient pressure by "plugging" the access ports of cavities is a recent example. This was done by wire bonding gold stud bumps directly into the access ports to an otherwise sealed and liquidfilled micro-cavity [4]. Deformation and cold welding of partly overlapping electroplated gold sealing rings between two wafers for room-temperature cavity formation and vacuum encapsulation has also been demonstrated [5]. Low-temperature deformation of gold is extensively used in a process called coining, where wire bonded gold bumps are plastically deformed in order to both make the top surface flat and to achieve a predetermined bump height. This process was originally introduced in order to increase the electrical and mechanical reliability of stud bumps used in flip chip packaging [6]. Recently it has also

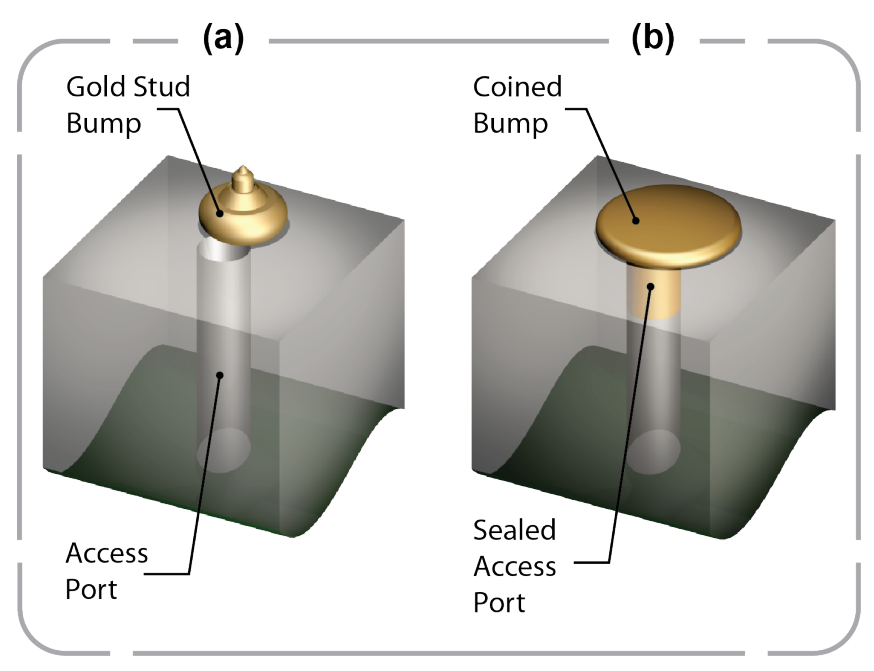

Figure 1: a) First, a wire bonded gold bump is placed partly overlapping the access port of a cavity. (b) Second, the bump is compressed in a vacuum atmosphere, causing plastic deformation and a flow of the bump material into the access port, thereby clogging the port and sealing the cavity.

been used for 3-dimensional micro structuring [7], where the bumps were imprinted with a structured mold.

In this paper we report on a novel wafer-level room temperature vacuum sealing process consisting of two steps. First, wire bonded gold bumps are placed in such a way that they overlap parts of the access ports of cavities. Second, a batch coining process is performed, causing mechanical deformation of the bump by applying a force that causes the bump to plastically deform and flow into the access ports, thereby clogging them as shown in Fig. 1. This is performed using the combination of a high-speed wire bonder and a commercial wafer bonder, ensuring a costefficient implementation with standard tools. Additional mechanical stabilization of the seal is not needed.

The placement of the bumps using a high-speed wire bonder can be a cost-efficient solution even for a large number of cavities since wire bonding is an extremely mature back-end technology with very high throughput [8]. For very high volume applications the cost of wire bonding processes has been reported to be on the order of 14 USD / 100,000 bumps [9]. The only prerequisites for using this method are small constrictions into the cavity, a certain stiffness of the package material and a wire-bondable surface. 
(a)
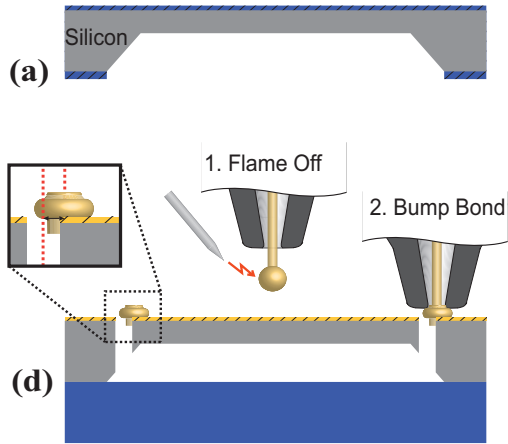

(b)
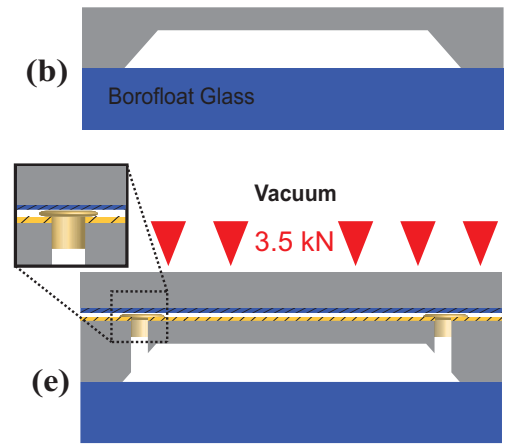

(c)

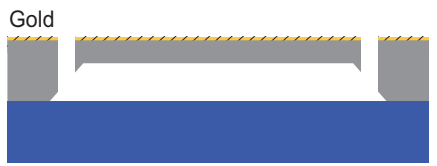

Figure 2: a) Recess formation by $\mathrm{KOH}$ etching of an oxide patterned double side polished silicon substrate. b) Wet strip of silicon dioxide layers and subsequent cavity formation by anodic bonding to a borofloat glass substrate. c) Lithography and deep reactive ion etching of the access ports. Subsequent gold sputter deposition. d) 1.) In the wire bonder, an electrical discharge locally melts the gold wire and forms a sphere at the end of the wire. 2.) The gold bump is bonded with an offset in order to not fully cover and clog the access port. e) A polished Si wafer is placed on the bumps and the stack is placed in a wafer bonder. At a chamber pressure of $10^{-5}$ mbar a bond force is applied, pressing the bumps into the holes and hermetically sealing them. $f$ ) The difference between the cavity pressure and the ambient pressure causes a bending of the silicon membrane.

\section{FABRICATION}

For a proof of concept, test cavities have been fabricated according to the process scheme depicted in Fig. 2. The vacuum cavity structures consist of a silicon cavity with access ports and a borofloat glass cap. The cavity wafer is a $550 \mu \mathrm{m}$ thick double-side polished $100 \mathrm{~mm}$ silicon substrate with a $2 \mu \mathrm{m}$ thick silicon oxide layer on both sides, created by thermal wet oxidization at $1100{ }^{\circ} \mathrm{C}$. The silicon dioxide acts as a hard mask for a wet etching step with $\mathrm{KOH}$, which forms the cavity on the backside of the substrate. The etch depth was $400 \mu \mathrm{m}$, which led to a membrane thickness of the cavities of approximately $150 \mu \mathrm{m}$ and a cavity volume of $50 \mu \mathrm{l}$. The silicon dioxide layer is subsequently removed on both sides by BHF etching in order to prepare the wafer for anodic wafer bonding to the $500 \mu \mathrm{m}$ thick borofloat cap substrate, as depicted in Fig. 2b. The anodic bonding was done using a $5 \mathrm{kN}$ tool force and a bias of $800 \mathrm{~V}$ in a vacuum atmosphere.

A standard lithography on the front-side of the substrate defines the circular openings for the access ports. The ports are intentionally placed above the tapered sidewall of the 111 crystal plane exposed by the $\mathrm{KOH}$ etch. This protects the fragile $12 \times 12 \mathrm{~mm}^{2}$ silicon membrane from being damaged by the wire bonding process, which is performed in a later step. As depicted in Fig. 2c, a Bosch DRIE process creates the access ports. Finally, a 100 $\mathrm{nm} \mathrm{TiW}+500 \mathrm{~nm} \mathrm{Au}$ sputter deposition on the frontside serves as adhesion layer for the subsequent sealing process. This metallization additionally covers the top of the side-walls of the access ports, which are still covered with the passivation polymer from the DRIE process.

The vacuum sealing was performed in two steps. First, gold bumps were wire bonded off-center on $30 \mu \mathrm{m}$ diam- eter access ports to otherwise enclosed cavities as illustrated in Fig. 2d. The gold bumps were bonded at a rate of 14 bumps/s with a fully automated ESEC 3100+ wire bonder (ESEC Ltd, Switzerland). The bumping process was optimized to obtain high and narrow bump shapes. The wire bonding tool is a thermo-compression type where temperature, force and ultrasonics are applied to bond the gold bumps to the gold layer on the substrate. Temperature is applied by a heated substrate chuck, ultrasonics and force by the bond head. An increased ultrasonic power and duration was used in order to optimize the bond process towards a low substrate temperature. Typical chuck temperatures of $100-160^{\circ} \mathrm{C}$ could thereby be decreased to 40 ${ }^{\circ} \mathrm{C}$ in order to maintain a low thermal budget throughout the whole sealing process.

The cavity wafer was finally transferred to a Süss CB8 substrate bonder (Süss MicroTec AG, Germany) where the cavities were batch sealed by compressing the bumps in a vacuum environment. Therefore, an oxidized silicon substrate was placed on top of the cavity wafer in order to ensure a flat and evenly distributed compression of the bumps over the entire wafer area. The wafer stack is placed in the bond chamber and after pumping to a vacuum pressure of $10^{-5}$ mbar, the wafer stack was compressed with a total force of $3.5 \mathrm{kN}$ from the bond tool as indicated in Fig. 2e. This bond force corresponds to a force per bump of $9 \mathrm{~N}$. The entire compression process was performed with the bond chucks at room temperature. The plastic deformation of the bumps caused the cavities to become hermetically sealed, as illustrated in Fig. 2e and f. The pressure difference between the cavity volume and the atmospheric pressure caused a bending of the membrane as indicated in Fig. 2f. This membrane bending was 


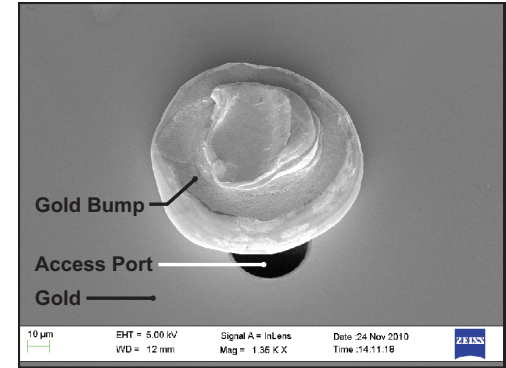

(a) A gold bump prior to vacuum sealing. The bump has a diameter of approximately $95 \mu \mathrm{m}$ and a height of $50 \mu \mathrm{m}$. It is placed with an offset of $35 \mu \mathrm{m}$ from the center of the 30 $\mu \mathrm{m}$ diameter port.

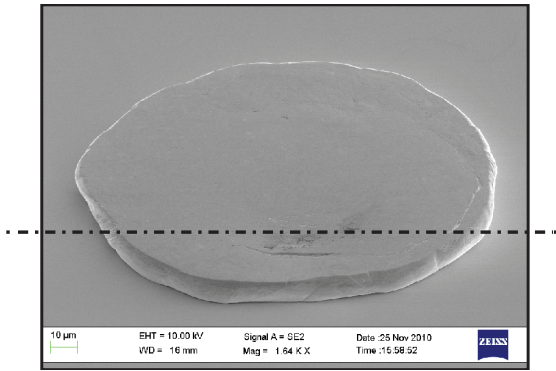

(b) A coined gold bump after the vacuum sealing. A force of approximately $9 N$ per bump was used to plastically deform the bumps and seal the access ports. The bump is flattened and its height is reduced to $15 \mu \mathrm{m}$.

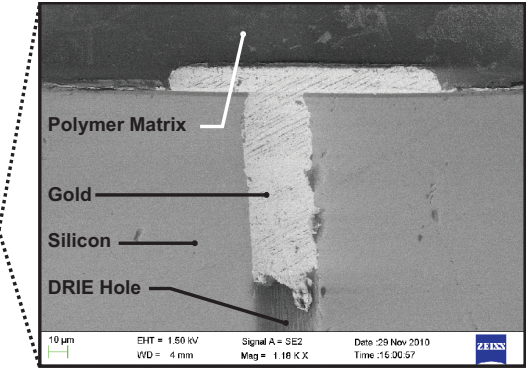

(c) Cross sectional view of a sealed access port. The cross section was made by grinding and polishing. The gold has been pressed $90 \mu \mathrm{m}$ into the access port.

Figure 3: SEM micrographs of wire bonded gold bumps before (a) and after (b-c) compression.

monitored over time in order to evaluate the hermeticity and leak rate of the seals.

\section{EXPERIMENTS AND RESULTS}

Fig. 3a shows an SEM micrograph of a gold bump that has been wire bonded with an offset with respect to the access port. The used wire bonding tool has a fully automated pattern recognition system, which is able to detect the access port and to place the stud bump with a predetermined offset to the center of the port. The specified placement accuracy is $2.5 \mu \mathrm{m}(3 \sigma)$. The effect of the plastic deformation is clearly visible when comparing the SEM pictures of the gold bump before, Fig. 3a, and after compression, Fig. 3b. The access port has been completely covered by the deformed bump and the top surface is flat. A cross sectional SEM view of a sealed access port is shown in Fig. 3c. This was made by encasing a package in a conductive polymer matrix followed by grinding and polishing carefully. The result shows that the compression caused the gold from the bump to completely fill and seal the access port to a depth of $90 \mu \mathrm{m}$. The maximal size of the access port is limited by the available volume of gold and the applied pressure for deformation. The gold ball size formed during flame-off limits the gold volume and hence the maximal port diameter during these experiments.

The membranes of the vacuum-sealed cavities deflected when exposed to atmospheric pressure. The magnitude of the deflection was measured by white light interferometry and corresponded to a cavity pressure of less than 10 mbar when compared to FEM simulations of a membrane model simulated using Comsol [10]. The measurement is indicative and not precise for this low pressure range. A typical deflection measurement is seen in Fig. 4, which shows the continuous surface deformation (darker region) caused by the membrane deformation of a vacuum sealed cavity with 80 sealed ports (bright spots).

The membrane deflection was measured in air over 5 days in order to evaluate the leak rate. In Fig. 5 the results of the deflection change for 4 cavities are compared to a membrane simulated using the atmospheric pressure variations measured at a close-by weather station. The change in membrane deflection has been normalized to the initial deflection of the membrane. Three of the four cavities have no detectable leak. The fourth device has a fine leak of $6 \times 10^{-8} \mathrm{mbarL} / \mathrm{s}$, when a linear relationship between the deformation and the pressure is assumed [11]. The deflection variations of the 3 sealed cavities follow the expected deflection simulated using the atmospheric pressure variations. The leak rate into the three sealed cavities

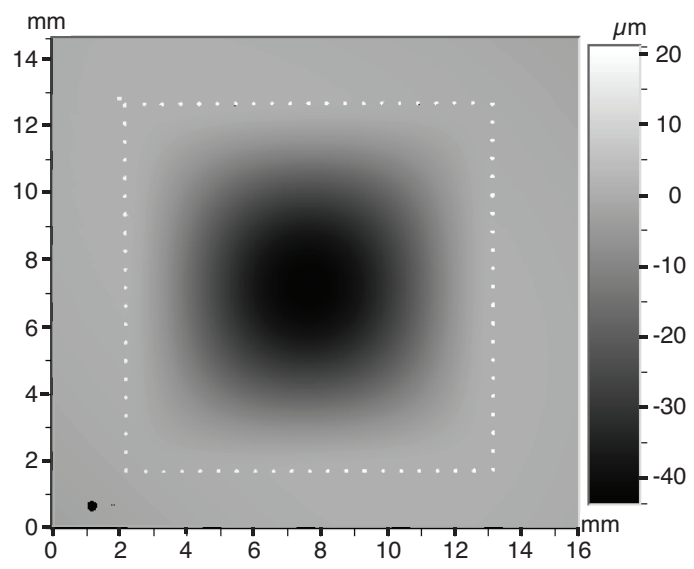

Figure 4: Profilometric image of a single sealed cavity with 80 access ports (bright spots), which were vacuum sealed by the presented method. The zero-level corresponds to the undeflected substrate surface. 


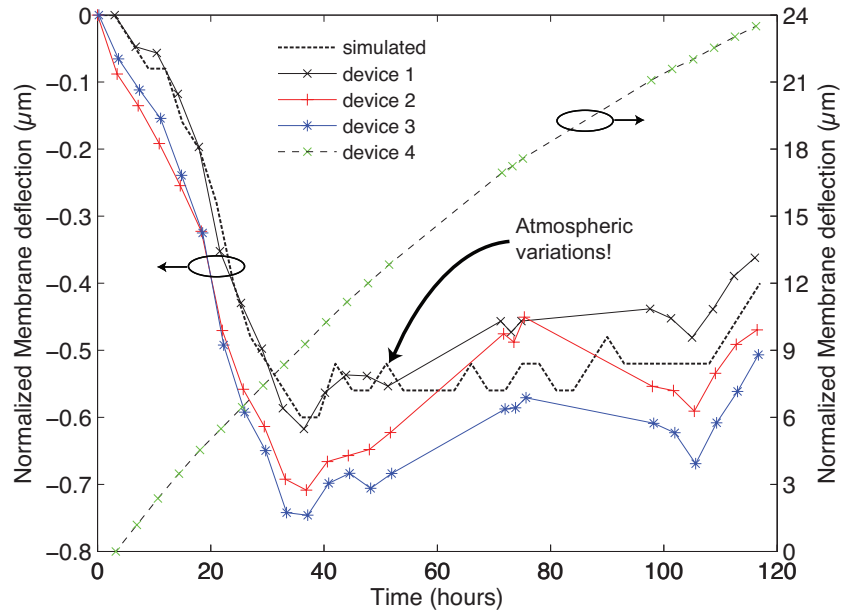

Figure 5: Measurement made with an optical profilometer of the membrane deflection over time in order to evaluate the sealing. 4 cavities are compared to a membrane simulated using the atmospheric pressure variations measured at a close-by weather station (dashed line). Three of the four cavities appear to be sealed and their curves have been normalized to their initial deflection. Device 4 has a fine leak of about $6 \times 10^{-8} \mathrm{mbarL} / \mathrm{s}$. The deflection variations of the sealed cavities fit well to the atmospheric pressure variations.

is below the limit of detection, which for this method under these circumstances is $6 \times 10^{-12} \mathrm{mbarL} / \mathrm{s}$ per sealed port [12].

\section{CONCLUSIONS}

We have described and demonstrated a novel room temperature vacuum sealing process using deformation of wire bonded "bumps" into access ports of preformed cavities. No leak was detected with the evaluation method, showing that the leak rate is smaller than $6 \times$ $10^{-12} \mathrm{mbarL} / \mathrm{s}$. This vacuum sealing method enables uncomplicated and cost efficient vacuum sealing at roomtemperature using standard commercial processing tools and processes.

\section{ACKNOWLEDGEMENTS}

This work was in part financed by the European Commission through the seventh framework program in the project xMEMs (267528).

\section{REFERENCES}

[1] M. Esashi, "Wafer level packaging of MEMS", J. Micromech. Microeng., vol 18, no 7, 073001, May 2008.

[2] S. N. Farrens, J. R. Dekker, J. K. Smith and B. E. Roberds, "Chemical free room temperature wafer to wafer direct bonding", J. Electrochem. Soc., vol. 142, no. 11, pp. 3949-3955, Nov. 1995.

[3] Y.-T. Cheng, W.-T. Hsu, K. Najafi, C. T.-C. Nguyen and L. Lin, "Vacuum packaging technology using localized aluminum/silicon-to-glass bonding", $\mathrm{J}$. Microeletrcomech. Syst., vol 11, no. 5, pp. 556-565, Oct. 2002.

[4] M. Antelius, A. Fischer, F. Niklaus, G. Stemme and N. Roxhed, "Hermetic integration of liquids in MEMS by room temperature, high-speed plugging of liquidfilled cavities at wafer level", Proc. IEEE Int. Conf. on Micro Electro Mechanical Systems (MEMS), Cancun, Mexico, Jan 23-27, 2011, pp. 356-359.

[5] A. Decharat, J. Yu, M. Boers, G. Stemme and F. Niklaus, "Room-temperature sealing of microcavities by cold metal welding", J. Microelectromech. Syst., vol. 18, no. 6, pp. 1318-1325, Oct. 2009.

[6] L. Levine, "Ball bumping and coining operations for TAB and Flip Chip", 3rd Int. Symp. on Advanced Packaging Materials. Proc., Braselton, USA, Mar. 912, 1997, pp. 110-112.

[7] R. S. Pai, M. M. Crain, and K. M. Walsh, "Maskless shaping of gold stud bumps as high aspect ratio microstructures", Microelectron. Eng., vol. 88, pp. 135139, Jan. 2011.

[8] G. Harman, Wire Bonding in Microelectronics, 2nd ed., McGraw-Hill Professional, 1997.

[9] Stud bumping and die attach for expanded flip chip applications, Advanced Packaging, September 2004.

[10] M. A. Hopcroft, W. D. Nix and T. W. Kenny, "What is the Young's modulus of silicon?", J. Microeletrcomech. Syst., vol 19, no. 2, pp. 229-238, Apr. 2010.

[11] M. Giovanni, Flat and Corrugated Diaphragm Design Handbook, Marcel Dekker Inc., New York, 1982.

[12] A. Goswami and B. Han, "On ultra-fine leak detection of hermetic wafer level packages", IEEE Trans. Adv. Packag., vol 31, pp. 14-21, Feb. 2008. 